\title{
Die Urämie als biochemisches Problem
}

\author{
von \\ HERMANn SARTORIUS \\ Aus der Medizinischen Universitäts-Poliklinik Freiburg. (Direktor: Prof. Dr. H. Sarre)
}

(Der Schriftleitung zugegangen am 27. November 1962)

\begin{abstract}
Die klinischen Symptome der Urämie sind durch tiefgreifende Störungen des gesamten Stoffwechsels gekennzeichnet. Eine einheitliche Substanz, die man als „urämisches Toxin“ bezeichnen könnte, ist bisher trotz jahrzehntelanger Forschung nicht gefunden worden. Neue Erkenntnisse werden möglicherweise durch das Dialyseverfahren gewonnen werden, da es durch Anwendung einer Kunststoffmembran gelingt, gewisse Metaboliten auszuschließen. Viele klinische Symptome der Urämie lassen sich im Tierexperiment mit Guanịdin nachahmen. Besondere Beachtung verdienen daneben Untersuchungen, die Enzymblockierungen durch Metaboliten des Eiweißstoffwechsels nachgewiesen haben.

In eigenen Untersuchungen werden die Störungen des Kohlenhydratstoffwechsels und des Säure-Basen-Haushaltes vor und nach Behandlung von urämischen Patienten mit der extrakorporalen Dialyse beschrieben.
\end{abstract}

The clinical symptoms of uraemia are characterised by deep-seated metabolic disturbances. A single substance, which could be called "uraemic toxin", has, despite decades of research, not been found. Progress will possibly be made by the dialysis method, since it is possible to exculde certain metabolites with a synthetic membrane. Many clinical symptoms of uraemia can be simulated in animal experiments with guanidine. Special consideration should also be given to studies which have shown the inhibition of enzymes by the products of protein metabolism. In our own work, disturbances of carbohydrate metabolism and of acid-base balance before and after treatment of uraemic patients with artificial, extracorporal dialysis are described.

Urämie bedeutet renale Dekompensation. Wie die Herzinsuffizienz kann eine Urämie durch eine große Anzahl von organischen Erkrankungen entstehen, die primär das Nierenparenchym befallen, den Harnabfluß durch Obstruktion der Harnwege behindern oder indirekt über ein Versagen des Kreislaufs die Nierenfunktion einschränken oder gar aufheben. So finden sich die klinischen und blutchemischen Symptome einer Urämie z. B. bei Schock, Septikämie, anhaltendem Erbrechen usw., also pathophysiologischen Zuständen, die keineswegs mit einer morphologischen Läsion der Nieren beginnen. Diese Vielzahl ätiologischer Faktoren hat bis heute eine einheitliche Definition der Úrämie erschwert. Aber auch der Versuch einer Zuordnung von klinischurämischen Symptomen zu den einzelnen tiefgreifenden blutchemischen Veränderungen war bisher nicht mit Sicherheit möglich. Jahrzehntelang haben Kliniker und Biochemiker nach einem urämischen Toxin gesucht, das imstande ist, allein das schwere klinische Zustandsbild der Intoxikation zu verursachen; aber die Worte FourNIERs aus dem Jahre 1863 blieben bis heute gültig: «Enfin, cette alteration chimique du sang est encore mal definie, et la science attend sur ce point de nouvelles recherches». Wir kennen zur Zeit keinen Metaboliten, dessen Retention allein für die urämische Intoxikation verantwortlich gemacht werden könnte. Nach unseren heutigen Kenntnissen ist Utämie vielmehr die komplexe Resultante einer Vielzahl von Reaktionen und Mechanismen, die durch eine Niereninsuffizienz in Gang kommen und unterhalten werden kann. Dennoch hat dieses Problem durch die Anwendung der Dialyse (sog. „künstliche Niere“) in den letzten Jahren wieder neue Anregungen erhalten. Durch die Dialyse ist es möglich, die Symptome der Urämie zu beseitigen. Damit steht aber fest, gleich, ob es sich nun um eine einheitliche oder verschiedene urämische Substanzen handelt, daß auf sie einige bestimmte chemisch-physikalische Kriterien zutreffen müssen: das Molekül passiert eine Porengröße von $30 \AA$, denn das ist die Porengröße des-Zellophans der Dialysiermembran. Sein Radius, gleich in welcher Projektion, muß bei dieser Porengröße durchtreten können. Es kann auch nicht asymmetrisch sein, da in der Zellophanmembran die Porenlänge von $80 \mu$ passiert werden muß. Seine Ionisation kann nicht erheblich sein, da es von der Membran weder abgestoßen noch angezogen wird. Auch eine Eiweißbindung entfällt, da die Substanz sonst nicht passager wäre, und seine Konzentration muß hoch genug sein, um einen signifikanten Gradienten während der Dialyse aufrechtzuerhalten. Unter Hinzuziehung dieser Indizien und mit neueren Arbeitsmethoden gelang es jüngst FrIMPTER und Trompson (1), einige konjugierte Aminosäuren zu identifizieren, die auch im urämischen Plasma nach Hydrolyse mit $6 n$ - $\mathrm{HCl}$ nachzuweisen waren. CoHeN und Mitarbeiter (2) nehmen an, daß retinierte Guanidine eine wichtige Rolle bei der Störung im Kohlenhydratstoffwechsel während einer Urämie spielen.

Harnstoff als Stoffwechselendprodukt aus dem Eiweißstoffwechsel und als der wesentliche Anteil der Rest-NFraktion wird hinsichtlich seiner Toxizität unterschiedlich beurteilt. Durch Harnstoffbelastung normaler Versuchspersonen kann seine Konzentration im Blut auf 
$100 \mathrm{mg} \%$ und mehr ansteigen, ohne daß urämische Symptome auftreten. SARRE (3) sowie MERRIL und Mitarb. (4) dialysierten urämische Patienten gegen Harnstofflösungen gleicher Konzentration und fanden nach Beendigung einer sechsstündigen Dialyse Symptomenfreiheit mit deutlicher Besserung des Allgemeinbefindens. Bollmann und MaNN (5) haben vor etwa 40 Jahren folgenden interessanten Tierversuch vorgenommen. Sie transplantierten die Ureteren eines Hundes in verschiedene Darmabschnitte. Das rektale Transplantat ergab nur.eine unwesentliche Steigerung aller Rest-N-Substanzen im Blut. Nach Einpflanzung der Ureteren in das Duodenum stiegen dagegen alle $\mathrm{N}$-Metaboliten im Blut rasch an, und das Versuchstier starb nach einigen Tagen. Wurden die Ureteren in das untere Ileum eingesetzt, so stieg anfänglich nur der Harnstoff im Blut an, und zwar bis zu einer Höhe von $800 \mathrm{mg} \%$, ohne daß Urämie-Symptome auftraten. Andere Untersucher fanden dagegen bei ihren klinischen Beobachtungen und Experimenten Zusammenhänge zwischen der Höhe des Harnstoffes im Blut und einigen urämischen Symptomen. Vorwiegend wurde über zentralnervöse Störungen berichtet. STEvenson und Mitarb. (6) infundierten Versuchstieren hochkonzentrierte Harnstofflösungen. Bei einem Blutspiegel von $300 \mathrm{mg} \%$ traten deutliche EEG-Veränderungen auf. Kontrollgruppen, bei denen die Osmolarität im Liquor entsprechend erhöht wurde, zeigten aber keine Veränderungen im EEG. Unverändert bei diesen Versuchen blieben auch Ammoniak-, Kreatinin- und Elektrolytkonzentrationen. Neueste Untersuchungsergebnisse haben gezeigt, daß auch schon bei niedrigeren Harnstoffkonzentrationen als in der Urämie eine Reihe von Enzymen blockiert wird. Kompetitiv gehemmt wurden durch Harnstoff: die Xanthin-Oxydase, die MilchsäureDehydrogenase, die saure Phosphatase, die Histidase, Thyrosinase und die 1-Amino-Oxydase. Nicht kompetitiv gehemmt: die Katalase, Rhodanase und anorganische Pyrophosphatase (7). Guanidine zeigten aber eine 10-80 fach stärkere Hemmung der genannten Enzyme.

Die Injektion von Harnstoff kann eine Hyperglykämie auslösen $(8,9)$. Quantitative Studien des Kohlenhydratstoffwechsels in der Urämie wurden schon vor Jahrzehnten durchgeführt. So berichtete NeUBaUER (10) bereits im Jahre 1910 über erhöhte Nüchternblutzucker (NBZ) bei nichtdiabetischen dekompensierten Nierenkranken. Der höchste Blutzucker wurde bei Urämikern festgestellt. PERKoFF und Mitarb. (9) haben anhand intravenöser Glukosetoleranzteste an nichtdiabetisćhen urämischen Patienten einen Kurvenverlauf nachweisen können, der einem leichten Diabetes mellitus gleichkommt. Eigene Untersuchungen (SARroRIUS und Piazolo (11)) an 16 akuten und chronischen Urämien ergaben, daß der Nüchternblutzucker mit ansteigendem Rest- $\mathrm{N}$ deutlich bis zu diabetischen Werten ansteigt (Abb. 1). Die Erhöhung des 2. Gipfels über dem ersten bei den Glukosedoppelbelastungen weist eine direkte Beziehung zur Höhe des Blut-Rest-N auf.
Auch die Untersuchungsergebnisse der Insulinbelastungen bei unseren urämischen Patienten waren diabetisch. Am empfindlichsten deuteten aber die Untersuchungsergebnisse mit Tolbutamid-Belastungen auf die diabetische Stoffwechsellage beim Urämiker. Die 20- und

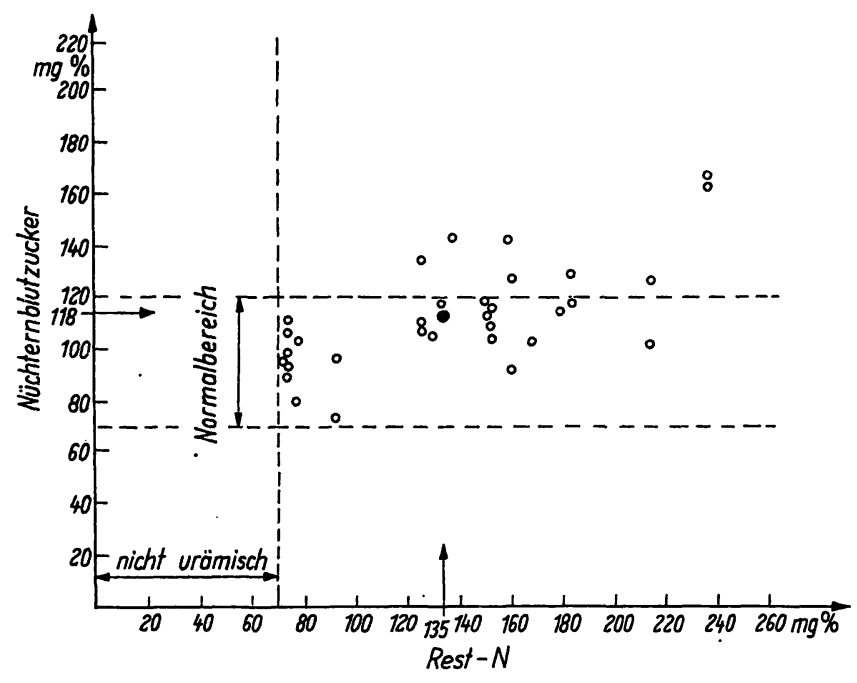

Abb. 1

Nüchternblutzucker (NBZ) und Rest-N-Werte im Blut von 32 Urämikern.

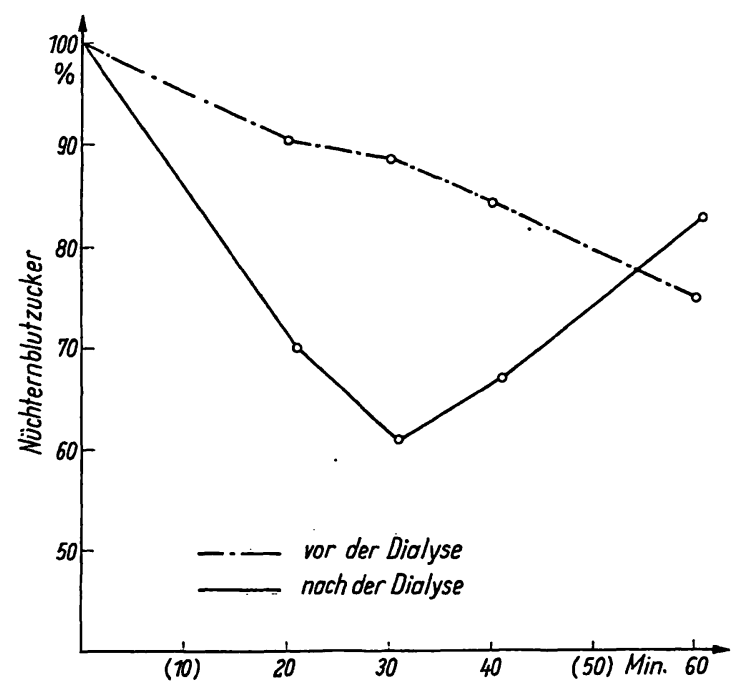

Abb. 2

Rastinonbelastungskurve vor und nach der künstlichen Niere. Völlige Normalisierung nach der Dialyse.

30-Min.-Werte des NBZ bei 12 urämischen Patienten wiesen alle eine diabetische Stoffwechsellage auf. Postdialytisch, mit einer Teilnormalisierung des Rest-N im Blut fand sich eine Normalisierung des Tolbutamidtestes (Rastinon-Test) (Abb. 2). Nach den kürzlich mitgeteilten Untersuchungsergebnissen von RAJAGOPALAN über die Hemmung von Enzymen durch den Harnstoff, wäre es möglich, daß in der Urämie durch die blockierende Wirkung von Harnstoff eine Glukoseverwertungsstörung auftritt. Fassen wir unsere derzeitigen Kenntnisse über die Wirkung des Harnstoffs zusammen, so zeigte sich in neuerer Zeit, daß Harnstoff direkt toxisch auf einige Organsysteme wirkt und darüber hinaus Enzymsysteme blockieren kann. 
Bei einer Anzahl von Patienten mit Urämie findet man immer wieder deutliche Diskrepanzen zwischen der Höhe des Blut-Harnstoffs und des Kreatiningebalts. Die Kreatininkonzentration entspricht meist $10 \%$ der Harnstoffkonzentration. Patienten mit akutem Nierenversagen und gleichzeitiger schwerer Lebererkrankung haben aber häufig höhere Kreatininwerte. Das gleiche gilt für junge muskelstarke Patienten mit akutem Nierenversagen und Niereninsuffizienz (CRUsH-Syndrom). Patienten mit hohem Serum-Kreatinin aber noch relativ niedrigem Harnstoffwert können aber schon typische urämische Symptome aufweisen. Hierzu ein Beispiel aus unserem Patientengut:

Ein 19jähriger Patient erlitt bei einem Mopedunfall eine Fraktur der linken Beckenschaufel mit einem großen flächenhaften Hämatom am Rücken und im Bereich des Beckens. Anschließend kam es zu einem akuten Nierenversagen. Die Nierenbiopsie ergab eine Nekrose des Nierenparenchyms mit teilweise randständig erhaltenem Gewebe. Der Patient mußte mehrfach während seine oligurischen Phase dialysiert werden, ehe die Diurese wieder einsetzte. Die Indikationen für die insgesamt fünf Dialysen wurden wegen der immer wieder rasch auftretenden urämischen Symptome notwendig. Den klinischen Symptomen parallel gingen stets die Hyperkaliämie und ein deutlich erhöhter Kreatininspiegel bei gleichzeitig größeren Schwankungen der Harnstoff-Konzentrationen. Kreatinin läßt sich nun durch die Dialyse nicht so gut auswaschen wie z. B. Harnstoff, so daß der höhere Kreatininwert einmal durch die geringere Abschöpfung der Dialyse und zum anderen durch Neubildung aus dem zerstörten Muskelgewebe infolge des Crusb-Syndroms bedingt war.

Die Bedeutung der Harnsäure als urämisches Toxin konnte bisher weder klinisch noch experimentell bewiesen werden. Die Dialysierfähigkeit der Harnsäure bei der Behandlung mit der künstlichen Niere ist gegenüber dem Harnstoff deutlich geringer. Trotz dieser schlechteren Diffusion der Harnsäure verlieren sich aber die urämischen Symptome nach sechsstündiger Dialyse. Harnsäure ist einer derjenigen Metaboliten, der den höchsten Konzentrationsgradienten zwischen Blut und Liquor aufweist. Wegen dieses normalerweise vorhandenen hohen Gradienten hat man angenommen, daß durch Zusammenbruch der Blut-Liquorschranke in der Urämie die Harnsäurekonzentration im Liquor steigt und ein Teil der zentralnervösen Symptome hierdurch ausgelöst wird. Diese Hypothese konnte aber bisher durch experimentelle Untersuchungen nicht bestätigt werden. BeCher (12) wollte eine Proportionalität urämischer Symptome mit der Retentionsgröße der Darmfäulnisprodukte (Xanthoprotein-Reaktion) nachweisen und legte besonderen Wert auf seine Feststellung, daß es erst dann zur vollausgebildeten Urämie komme, wenn diese Substanzen schließlich in den Liquor übertreten. Wir können heute annehmen, daß der Retention von Phenolkörpern diese Bedeutung in der Pathogenese der Urämie wohl nicht zukommt. Durch die Dialyse gelingt es, die urämischen Symptome zu beseitigen. Dabei hat sich aber gezeigt, daß die in der Xanthoproteinreaktion erfaßten Phenolkörper durch die Zellophanmembran schlecht diffundieren, wahrscheinlich, weil die Phenole an Eiweiß gebunden sind.

Zusammensetzung und Menge der Plasma-Aminosäuren beim urämischen Patienten zeigen Abweichungen vom
Normalen. SalisBury und Mitarb. (13) untersuchten die freien und gesamten Aminosäuren bei urämischen Patienten am enteiweißten Plasma. Sie fanden Glykokoll, Arginin und Prolin deutlich erhöht, während andere Aminosäuren erniedrigt waren. $\mathrm{Zu}$ ähnlichen Ergebnissen kamen Doolan (14) und LeVenson (15). Übereinstimmend konnte aber festgestellt werden, daß qualitative und quantitative Veränderungen des PlasmaAminosäurespektrums nicht mit der.Intensität und dem Schweregrad der Urämie parallel gingen. Auch nach mehrfachen Dialysen bei einem Patienten mit akutem Nierenversagen und einer Oligurie-Phase von insgesamt 149 Tagen zeigte das Aminosäuremuster im Plasma starke Abweichungen von normalen (16). Bisher gelang es nicht nachzuweisen, daß dem Anstieg einzelner Aminosäuren im Plasma von Urämikern ein toxischer Effekt zuzuschreiben wäre. Wohl aber stellten SCHREINER und MAHER fest, daß die Infusion eines Proteinhydrolysates bei Patienten mit Urämie zu deutlicher Verschlechterung mit Übergang in Bewußtlosigkeit und eklamptischen Symptomen führte.

Guanidin und Indikan sind während einer Urämie im Blut erhöht. Guanidin wurde bereits 1915 von Foster (17) aus urämischem Blut extrahiert und als toxische Base erkannt, da es - Meerschweinchen injiziert - zu Konvulsionen, Koma und Tod der Versuchstiere führte. Allerdings gelang es bisher nicht, Guanidin in kristalliner Form aus urämischen Blut zu gewinnen. Nach diesen ersten Versuchen von Foster haben viele Nachuntersucher einen erhöhten Guanidinspiegel beim Urämiker gefunden. Unter den bisher bekannten chemischen Substanzen, die im urämischen Blut gefunden wurden, nimmt das Guanidin insofern eine Sonderstellung ein, als es durch Guanidin experimentell gelingt, viele urämische Symptome hervorzurufen und auszulösen. Dieser toxische Effekt von Guanidin kann durch Calcium und seltsamerweise auch durch Harnstoff abgeschwächt werden. $\mathrm{Da}$ man jetzt erst festgestellt hat, daß das Guanidin bedeutend intensiver Fermente blockiert als Harnstoff, wird ihm schon etwas voreilig der Rang des einen Urämietoxins zuerkannt. Mit ausreichender Sicherheit wird sich dieses interessante Problem aber erst dann beantworten lassen, wenn neuere und exaktere Bestimmungsmethoden für Guanidin ausgearbeitet sind. Über Guanidin-Konzentrationen im Blut vor und nach Dialyse liegen zur Zeit noch keine Angaben vor.

Dem Indikan kommt sicherlich keine wesentliche Bedeutung als Urämiestoff zu. Man darf vor allem nicht vergessen, daß $z u$ den Begleiterkrankungen einer Urämie Gastro-Entero-Kolitiden und manchmal ein paralytischer Ileus gehört, so daß gestörte Darmfunktion und intestinale Florà ursächlich für die erhöhten Indikankonzentrationen eine wichtige Rolle spielen. Im Zeitalter der Antibiotika, die häufig genug auch während einer Niereninsuffizienz angewendet werden, muß man in der Beurteilung von Toxikämien durch Indikanerhöhung vorsichtig sein.

Durch die Behandlung mit (der künstlichen Niere werden aber nicht nur die retinierten Metaboliten aus 
dem Eiweißstoffwechsel bei Urämikern entfernt, sondern es werden auch Störungen im Säure-Basen- und Elektrolythaushalt ausgeglichen. Bei chronischer und akuter Niereninsuffizienz mit begleitender Urämie kommt es fast stets zu einer metabolischen Azidose. Diese Azidose bei Urämie infolge Niereninsuffizienz kommt durch Sistieren der Austauschvorgänge von $\mathrm{H}^{+}$-Ionen gegen $\mathrm{Na}^{+}$-Ionen im distalen Tubulus und einer verminderten Bikarbonat-Einsparung zustande. Phosphate und Sulfate werden ungenügend ausgeschieden. Die ,unbestimmbare" Restfraktion auf der Anionenseite nimmt zu, das Bikarbonat ab. Wenn die kompensatorischen Vorgänge ausreichen, bleibt das extrazelluläre $\mathrm{p}_{\mathrm{H}}$ unverändert, und die Azidose ist kompensiert. Sinkt das $\mathrm{p}_{\mathrm{H}}$ aber unter 7,4, liegt eine dekompensierte metabolische Azidose vor. Bei einer Anzahl mehrfach dialysebehandelter Patienten fand sich eine metabolische Azidose. Diese Azidose ist kei cen urämischen Patienten durch Messung des Standard-Bikarbonats, der Puffer-Base, des aktuellen Bikarbonats und des aktuellen, arteriellen $\mathrm{p}_{\mathbf{H}}$-Wertes feststellbar (vgl. dazu Astrup (18)). Bei einer Gruppe von urämischen Patienten variierte das $\mathrm{p}_{\mathrm{H}}$ vor der Dialyse von 7,27-7,54. Im Verlauf der Dialysen stieg das extrazelluläre $\dot{p}_{\mathrm{HI}}$ an und gelangte in den Normalbereich und darüber hinaus (Abb. 3). Aus der gleichen Behandlungsgruppe lagen die Werte für das Standardbikarbonat ebenfalls unterhalb des Normalbereiches. Am Ende der Dialysen erreichte das Standardbikarbonat in 7 Fällen den Normbereich (Abb. 4). Der Kohlensäurepartialdruck, der zu Beginn der Dialyse bei allen Patienten mit metabolischer Azidose erniedrigt war - und zwar als Ausdruck einer respiratorischen Kompensation -, änderte sich während der Dialyse nur geringfügig, so $\mathrm{da} \beta$ es am Ende der Dialyse zu einer respiratorischen Alkalose kam (Abb. 5). Daß die primäre metabolische Azidose durch die Behandlung mit der Dialyse behoben wird, beruht auf einer Erhöhung der Bikarbonatkonzentration im Blut des Patienten. Die Azidose beim Urämiker führt gleichzeitig zu Elektrolytverschiebungen zwischen Extra- und Intrazellulärraum (EZR u. IZR).

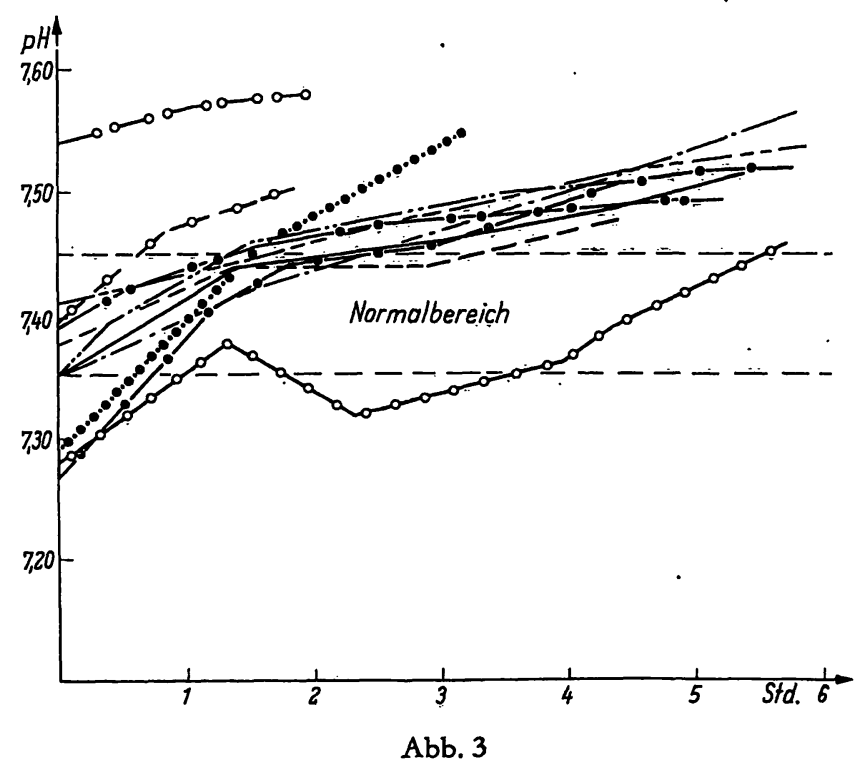

Anderung des aktuellen pH-Wertes während der Dialyse.

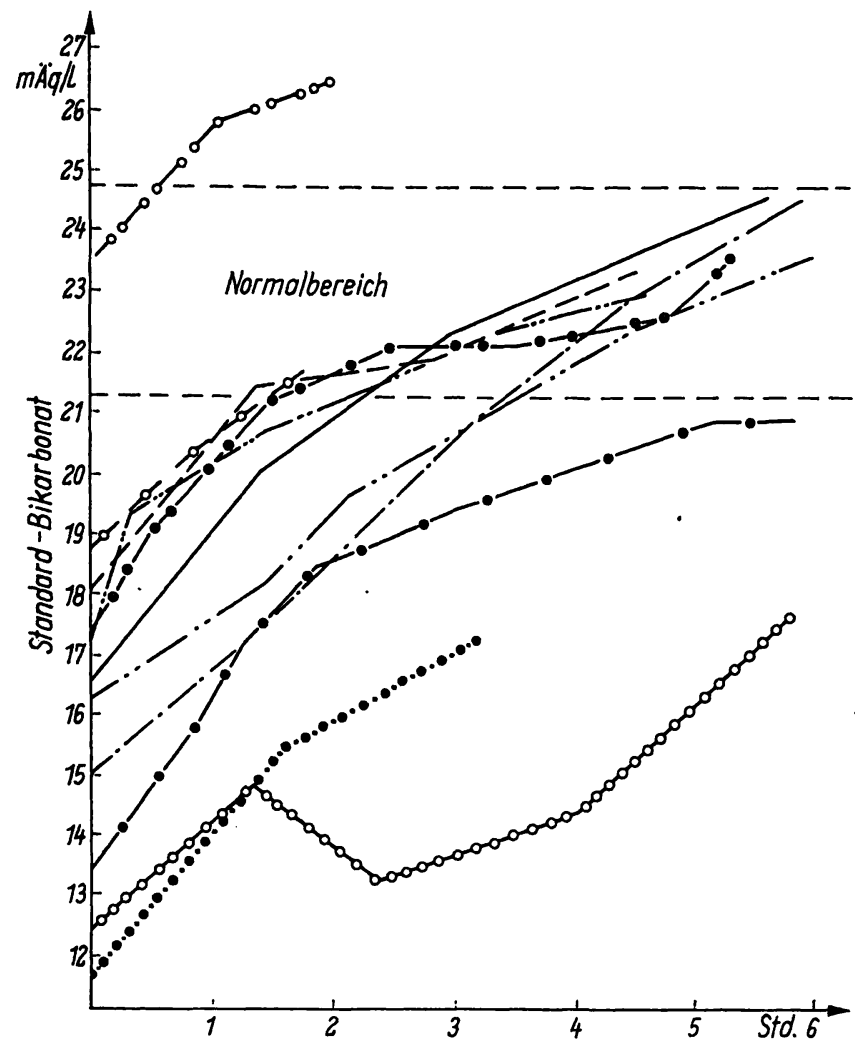

Abb. 4

Anderung von Standard-Bikarbonat (mval/l) während der Dialyse.

$p$

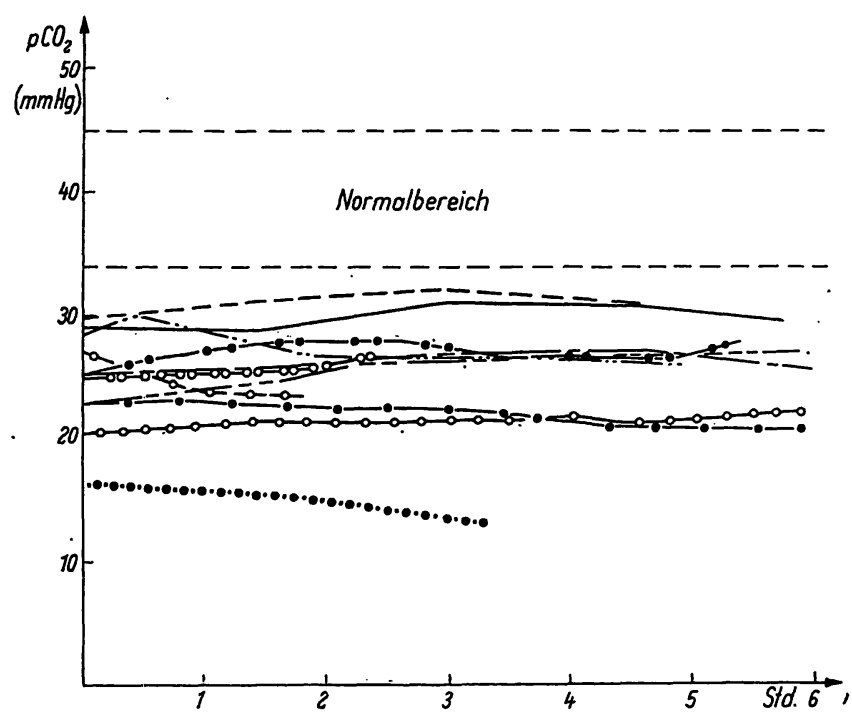

Abb. 5

Der arterielle Kohlensäurepartialdruck während der Dialyse.

Davon werden in erster Linie Natrium und Kalium betroffen. Diese Elektrolytverschiebungen betreffen aber nicht nur Kalium und Natrium, sondern gleichzeitig treten auch Phosphat, Sulfat sowie Magnesium aus der Zelle in den Extrazellulärraum über und Chlorund Wasserstoffionen in die Zelle ein. Im Plasma als einem Teil des EZR kann es deswegen bei einer akuten - aber auch im terminalen Zustand einer chronischen Urämie zur Hyperkaliämie und Hypermagnesiämie kommen. 
Magnesium setzt die Erregbarkeit der quergestreiften Muskulatur herab und hemmt alle Funktionen des Zentralnervensystems. Erhöht man die Plasma-Magnesium-Konzentration auf das Doppelte, so kommt es zum Auftreten von typischen urämischen Symptomen bis zum präkomatösen Zustand mit Blutdruckabfall.

Ähnliche Vergiftungszustände werden auch durch Kalium hervorgerufen. Der Hyperkaliämie mit Kaliumintoxikation kommt wohl bei der akuten Urämie als Störung im Elektrolythaushalt die größte Bedeutung zu. Das dominierende klinische Symptom der Kaliumintoxikation wie auch die der begleitenden Hypermagnesiämie ist die Wirkung auf das Reizleitungssystem des Herzens. Die mechanische Leistungsfähigkeit der Herzmuskulatur ist aufs engste mit dem Kaliumstoffwechsel des Herzmuskels verknüpft. Die Kaliumintoxikation ist aber nicht nur von der Höhe einer bestimmten Kalium-Konzentration im Plasma abhängig, sondern wird durch den gleichzeitig synergistisch wirksamen Magnesiumspiegel und die Azidose selbst sowie den antagonistisch wirksamen Natrium- und CalciumSpiegel mitbestimmt. Das EKG reflektiert die Elektrolytkonzentrationsänderungen während einer Urämie in ihrer Wirkung auf das Reizleitungssystem des Herzens. Es ist insofern als Resultante zwischen den verschiedenen Elektrolytkonzentrationen des Plasmas zu betrachten. (SARTORIUS (19)). Ist aber bei einer chronischen Niereninsuffizienz mit Urämie die Diarese noch ausreichend, so kann es trotz Azidose und Elektrolytverschiebungen auch zu einer Hypokaliämie kommen, da die tubuläre Kaliumsekretion bei der chronischen Nephritis bis in das Stadium der Pseudonormalurie ausreichend bzw. überschießend sein kann.

Die Elektrolytverschiebungen bei der akuten wie bei der chronischen Urämie können zur Hypocblorämie und Hyponatriämie führen. Verstärkt wird diese Saloprivie durch Kochsalzverlust im Harn und durch Erbrechen. Die bei chronischer Niereninsuffizienz gestörte Tubulusfunktion vermag Natriumionen nicht mehr gegen $\mathrm{H}^{+}$-Ionen auszutauschen. Der dadurch erfolgte Kationenverlust kann per se zu einer Azidose führen. Die osmotische Diurese macht sich gleichzeitig durch Wasserverlust und konsekutive Exsikkose bemerkbar. Erbrechen und Durchfälle verursachen weitere Demineralisation. Diese Wasser- und Salzverluste bei Demineralisation und Transmineralisation führen zu einem Anstieg der harnpflichtigen Stoffe. So kann sich auf die vorhandene organische Niereninsuffizienz zusätzlich eine saloprive Niereninsuffizienz aufpfropfen. Unter den gegebenen Umständen kann über einen circulus vitiosus der Urämietod beschleunigt eintreten. Elektrolytverschiebungen sind also direkte Folgen der gestörten Nierenfunktion mit Niereninsuffizienz. Die Entwicklung einer Urämie bei chronischer Niereninsuffizienz führt meist über einen Elektrolytverlust (Demineralisation) zur Azidose-Elektrolytverschiebung zwischen IZR und EZR (Transmineralisation), während die Urämie bei akutem Nierenversagen ohne vorangegangene Elektrolytverluste direkt mit Elektrolytverschiebungen durch Azidose-Einwirkung einsetzt.

Im urämischen Endstadium einer chronischen Niereninsuffizienz wie auch eines akuten Nierenversagens mit Urämie kommt es meist zur Überwässerung des $\mathrm{Pa}$ tienten. Bei solchen Patienten findet sich häufig eine herabgesetzte Osmolarität im EZR. Das bedeutet, daß der Verdünnungseffekt des retinierten Lösungsmittels die Retention der gelösten Stoffe übertrifft. Zusätzlich zur Flüssigkeitsausscheidungsstörung können noch innerhalb 24 Stunden größere Mengen neugebildeten Oxydationswassers auftreten, die bis zu $300 \mathrm{ml}$ pro $\mathrm{m}^{2}$ Körperoberfläche betragen können. Häufig genug kommt es zu Ödembildung. Drohende klinische Komplikationen sind dann immer Lungen- und Hirnödem.

Alle Störungen im Säure-Basen-, Elektrolyt- und Wasserhaushalt lassen sich durch die Dialyse beseitigen. Die Störungen im Elektrolythaushalt werden schnell kortigiert, weil Kationen und Anionen die größten Diffusionskonstanten besitzen.

\section{Literatur}

1. Frimpter, G. W. und D. Thompson, J. Clin. Invest. 39, 987 (1960). - 2. Cohen, B. D., N. Spritz, A. L. Rubin und E. H., Luckey, Clin. Res. 8, 227 (1960). - 3. Sarre, H., Verhdlg. Dtsch. Ges. Inn. Med. 269, 65 (1959). - 4. MerriL, J. P., M. Legrain und R. Hoigne, Amer. J. Med. 14,519 (1953). - 5. BollmanN, J. L. und F. C. ManN, Proc. Soc. exp. Biol. Med. 24, 923 (1927). - 6. Stevenson, G. C., R. C. Jacobs, M. W. Ross, W. F. Collins und C. T. Randt, Amer. J. Physiol. 197, 141 (1959). 7. Rajagopalan, K. V., I. Fridovich und P. Handler, Fed. Proc. 19, 49 (1960). - 8. Beaton, J. R. und J:Hunter, Fed. Proc. 19, 178 (1960). - 9. Perkoff, G. T., C. L. Thomas, J. D. Newton, J. C. Sellmann und F. H. Tyler, Diabetes N. Y. 7, 375 (1958). -
10. Neubauer, E., Biochem. Z. 25, 284 (1910). - 11. Sartorius, H. und P. Piazolo, Im Druck. - 12. Becher, E., Nierenkrankheiten, 1. Bd. Gustav Fischer, Jena (1947). - 13. Salisbury, P. F., M. S. Dunn und E. A. Murphy, J. Clin. Invest. 36, 1227 (1957). 14. Doolan, P. D., H. A. Harper, R. A. Watten, M. E. Hutchin und R. O. Canada, Trans. Amer. Soc. Artificial Internal Organs, 2, 113 (1956). - 15. Levenson, S., Ross Laboratories, 78-86 (1957). - 16. Schreiner, G. E. und J. F. Maher, Uremia, Charles Thomas (1960). - 17. Foster, N. B., Trans. Ass. Amer. Physicians 30, 305 (1915). - 18. Astrup, P., Klin. Wschr. 15, 749 (1957). - 19. Sartorius, H., 1. Symp. Ges. Nephr. 125. Georg Thieme (Stuttgart), (1961).

Priv.-Doz. Dr. med. Hermann Sartorius Medizinische Universitäts-Poliklinik 7 Freiburg i. Br., Hermann-Herder-Str. 6 